\title{
The Sparsity of Underdetermined Linear System via $l_{p}$ Minimization for $0<p<1$
}

\author{
Haiyang Li, ${ }^{1}$ Jigen Peng, ${ }^{2}$ and Shigang Yue ${ }^{3}$ \\ ${ }^{1}$ School of Science, Xi'an Polytechnic University, Xian 710048, China \\ ${ }^{2}$ Department of Mathematics, Xian Jiaotong University, Xian 710049, China \\ ${ }^{3}$ School of Computer Science, University of Lincoln, Lincoln LN6 7TS, UK \\ Correspondence should be addressed to Haiyang Li; fplihaiyang@126.com
}

Received 7 November 2014; Accepted 14 April 2015

Academic Editor: Kacem Chehdi

Copyright (C) 2015 Haiyang Li et al. This is an open access article distributed under the Creative Commons Attribution License, which permits unrestricted use, distribution, and reproduction in any medium, provided the original work is properly cited.

\begin{abstract}
The sparsity problems have attracted a great deal of attention in recent years, which aim to find the sparsest solution of a representation or an equation. In the paper, we mainly study the sparsity of underdetermined linear system via $l_{p}$ minimization for $0<p<1$. We show, for a given underdetermined linear system of equations $A_{m \times n} X=b$, that although it is not certain that the problem $\left(P_{p}\right)$ (i.e., $\min _{X}\|X\|_{p}^{p}$ subject to $A X=b$, where $0<p<1$ ) generates sparser solutions as the value of $p$ decreases and especially the problem $\left(P_{p}\right)$ generates sparser solutions than the problem $\left(P_{1}\right)$ (i.e., $\min _{X}\|X\|_{1}$ subject to $A X=b$ ), there exists a sparse constant $\gamma(A, b)>0$ such that the following conclusions hold when $p<\gamma(A, b)$ : (1) the problem $\left(P_{p}\right)$ generates sparser solution as the value of $p$ decreases; (2) the sparsest optimal solution to the problem $\left(P_{p}\right)$ is unique under the sense of absolute value permutation; (3) let $X_{1}$ and $X_{2}$ be the sparsest optimal solution to the problems $\left(P_{p_{1}}\right)$ and $\left(P_{p_{2}}\right)\left(p_{1}<p_{2}\right)$, respectively, and let $X_{1}$ not be the absolute value permutation of $X_{2}$. Then there exist $t_{1}, t_{2} \in\left[p_{1}, p_{2}\right]$ such that $X_{1}$ is the sparsest optimal solution to the problem $\left(P_{t}\right)\left(\forall t \in\left[p_{1}, t_{1}\right]\right)$ and $X_{2}$ is the sparsest optimal solution to the problem $\left(P_{t}\right)\left(\forall t \in\left(t_{2}, p_{2}\right]\right)$.
\end{abstract}

\section{Introduction}

Recently, considerable attention has been paid to the following sparsity problem. Given a full-rank matrix $A$ of size $m \times n$ with $m \ll n, m$-vector $b$, and knowing that $b=A X^{*}$, where $X^{*} \in \mathbf{R}^{n}$ is an unknown sparse vector, we expect to recover $X^{*}$. Although the system of equations is underdetermined and hence it is not a properly posed problem in linear algebra, sparsity of $X^{*}$ is a very useful priority that sometimes allows unique solution. Accordingly, one naturally proposes to use the following optimization model $\left(P_{0}\right)$ to obtain the sparsest solutions:

$$
\begin{aligned}
\left(P_{0}\right) \min _{X} & \|X\|_{0} \\
\text { s.t. } & A X=b,
\end{aligned}
$$

where $\|X\|_{0}$ denotes the number of nonzero components of $X$ (we call $\|\cdot\|_{0} l_{0}$ norm). This is one of critical problems in compressed sensing research. This problem is motivated by data compression, error correcting codes, $n$-term approximation, and so forth (see, e.g., [1]). It is known that the problem $\left(P_{0}\right)$ needs nonpolynomial time to solve (cf. [2]). It is crucial to recognize that one natural approach to tackle $\left(P_{0}\right)$ is to solve the following convex minimization problem:

$$
\begin{aligned}
\left(P_{1}\right) \min _{X} & \|X\|_{1} \\
\text { s.t. } & A X=b,
\end{aligned}
$$

where $\|X\|_{1}=\sum_{i=1}^{n}\left|x_{i}\right|$ is the standard $l_{1}$ norm. The study of this problem $\left(P_{1}\right)$ was pioneered by Donoho, Candès, and their collaborators and many researchers have made a lot of contributions related to the existence, uniqueness, and other properties of the sparse solution as well as computational algorithms and their convergence analysis to tackle the problem $\left(P_{0}\right)$ (see survey papers in [3-5]). However, the solutions to the problem $\left(P_{1}\right)$ are often not as sparse as those to the problem $\left(P_{0}\right)$. It is definitely imperative and required for many applications to find solutions which are more sparse 
than that to the problem $\left(P_{1}\right)$. A natural try for this purpose is to apply the problem $\left(P_{p}\right)(0<p<1)$, that is, to solve the following model:

$$
\begin{aligned}
\left(P_{p}\right) \min _{X} & \|X\|_{p}^{p} \\
\text { s.t. } & A X=b,
\end{aligned}
$$

where $\|X\|_{p}^{p}=\sum_{i=1}^{n}\left|x_{i}\right|^{p}$ (we call $\|\cdot\|_{p} l_{p}$-norm, though it is no longer norms for $p<1$ as the triangle inequality is no longer satisfied). Obviously, the problem $\left(P_{p}\right)$ is no longer a convex optimization problem. This minimization is motivated by the following fact:

$$
\lim _{p \rightarrow 0_{+}}\|X\|_{p}^{p}=\|X\|_{0}
$$

This model was initiated by [6] and many researchers have worked on this direction $[1,2,7-16]$. They demonstrate that (1) for a Gaussian random matrix $A$, the restricted $p$-isometry property of order $s$ holds if $s$ is almost proportional to $m$ when $p \rightarrow 0_{+}$(cf. [8]); (2) when $\delta_{2 s}<1$ (or $\delta_{2 s+1}<$ $\left.1, \delta_{2 s+2}<1\right)$, the optimal solution to the problem $\left(P_{p}\right)$ is the same as the optimal solution to the problem $\left(P_{0}\right)$ when $p>0$ small enough, where $\delta_{2 s}<1$ is the restricted isometry constants of matrix $A$ (similar for $\delta_{2 s+1}<1$, $\delta_{2 s+2}<1$ ) (cf. $[7,10,13]$ ); and (3) the $l_{p}$ minimization can be applied to a wider class of random matrices $A$ (cf. [11]). In addition, in $[7,15]$, the authors show that the problem $\left(P_{p}\right)$ generates sparser solution than the problem $\left(P_{1}\right)$ and the problem $\left(P_{p}\right)$ generates sparser solution as the value of $p$ decreases by taking phase diagram studies with a set of experiments. Nevertheless, are the conclusions showed by taking phase diagram studies true in theory? In the paper, we will answer this question by studying the sparsity of $l_{p}$ minimization. Firstly, using Example 2 we show, in general, that the answer to the question above is negative. Secondly, although the answer to the question above is negative, we can prove that, for a given underdetermined linear system of equations $A_{m \times n} X=b$, there exists a constant $\gamma(A, b)>0$ (we call it sparsity constant) such that the following conclusions hold when $p<\gamma(A, b)$.

(1) The problem $\left(P_{p}\right)$ generates sparser solution as the value of $p$ decreases (Theorem 7).

(2) Let $X_{p}$ be the sparsest optimal solution to the problem $\left(P_{p}\right)$. Then $X_{p}$ is the unique sparsest optimal solution to the problem $\left(P_{p}\right)$ under the sense of absolute value permutation (Corollary 6).

(3) Let $X_{1}$ and $X_{2}$ be the sparsest optimal solution to the problem $\left(P_{p_{1}}\right)$ and problem $\left(P_{p_{2}}\right)\left(p_{1}<p_{2}\right)$, respectively, and let $X_{1}$ not be the absolute value permutation of $X_{2}$. Then there exist $t_{1}, t_{2} \in\left[p_{1}, p_{2}\right]$ such that $X_{1}$ is the sparsest optimal solution to the problem $\left(P_{t}\right)\left(\forall t \in\left[p_{1}, t_{1}\right]\right)$ and $X_{2}$ is the sparsest optimal solution to the problem $\left(P_{t}\right)\left(\forall t \in\left(t_{2}, p_{2}\right]\right)$ (Theorem 8).

\section{The Sparsity of Underdetermined Linear System via $l_{p}$ Minimization}

Let $\mathscr{X}$ be the set of all solutions to the underdetermined linear systems $A X=b$. For the convenience of account, we call $X_{1}$ the absolute value permutation of $X_{2}$, which means that $\left(\left|x_{11}\right|,\left|x_{12}\right|, \ldots,\left|x_{1 n}\right|\right)$ is the permutation of $\left(\left|x_{21}\right|,\left|x_{22}\right|, \ldots,\left|x_{2 n}\right|\right)$, where $X_{1}=\left(x_{11}, x_{12}, \ldots, x_{1 n}\right)^{T}$ and $X_{2}=\left(x_{21}, x_{22}, \ldots, x_{2 n}\right)^{T} \in \mathscr{X}$.

Lemma 1 (see [17]). The problem $\left(P_{1}\right)$ may have more than one solution. Nevertheless, even if there are infinitely many possible solutions to this problem, we can claim that (1) these solutions are gathered in a set that is bounded and convex, and (2) among these solutions, there exists at least one with at most $m$ nonzeros.

The following example shows that, in general, it is not certain that the problem $\left(P_{p}\right)$ generates sparser solution than the problem $\left(P_{1}\right)$ and the problem $\left(P_{p}\right)$ generates sparser solution as the value of $p$ decreases.

Example 2. We consider the underdetermined linear system of equations $A X=b$, where

$$
A=\left(\alpha_{1}, \alpha_{2}, \alpha_{3}, \alpha_{4}\right)=\left(\begin{array}{rrrr}
-\frac{20}{29} & 1 & \frac{31}{87} & 0 \\
0 & 1 & \frac{8}{15} & 1 \\
\frac{60}{29} & 0 & \frac{463}{435} & -1
\end{array}\right)
$$

$b=(1,2,3)^{T}$. By Lemma 1 , the $l_{0}$-norm of the optimal solutions to the problem $\left(P_{1}\right)$ are not more than 3 , and hence the optimal solution is one of the following feasible solutions:

$$
\begin{aligned}
& \text { (1) } X_{1}=(0,-4 / 27,29 / 9,58 / 135)^{T} \text {; } \\
& \text { (2) } X_{2}=(0.1,0,3,0.4)^{T} ; \\
& \text { (3) } X_{3}=(1.45,2,0,0)^{T} ; \\
& \text { (4) } X_{4}=(1.45,2,0,0)^{T} .
\end{aligned}
$$

Furthermore, we can show that the optimal solution to the problem $\left(P_{p}\right)(p=0.8,0.95)$ is one of above feasible solutions. It is easy to calculate that

$$
\begin{aligned}
& \left\|X_{1}\right\|_{0.8}^{0.8}=3.2756, \\
& \left\|X_{2}\right\|_{0.8}^{0.8}=3.0472, \\
& \left\|X_{3}\right\|_{0.8}^{0.8}=\left\|X_{4}\right\|_{0.8}^{0.8}=3.0873,
\end{aligned}
$$




$$
\begin{aligned}
\left\|X_{1}\right\|_{0.95}^{0.95} & =3.6502, \\
\left\|X_{2}\right\|_{0.95}^{0.95} & =3.3706 \\
\left\|X_{3}\right\|_{0.95}^{0.95} & =\left\|X_{4}\right\|_{0.95}^{0.95}=3.3552, \\
\left\|X_{1}\right\|_{1} & =3.7999 \\
\|X\|_{1} & =3.5 \\
\left\|X_{3}\right\|_{1} & =\left\|X_{4}\right\|_{1}=3.45 .
\end{aligned}
$$

Thus $X_{2}$ is the optimal solution when $p=0.8$ and $X_{3}$ is the optimal solution when $p=0.95$ and $p=1$. However, $\left\|X_{2}\right\|_{0}=$ $3,\left\|X_{3}\right\|_{0}=2$. Therefore, the problem $\left(P_{p}\right)$ does not generate sparser solution than the problem $\left(P_{1}\right)$ and the problem $\left(P_{p}\right)$ does not generate sparser solution as the value of $p$ decreases.

In the following, we will prove the conclusions mentioned in Introduction.

We define two functions $f(t)=\|X\|_{t}=\left(\left|x_{1}\right|^{t}+\cdots+\right.$ $\left.\left|x_{k}\right|^{t}\right)^{1 / t}(t>0)$ and $g(t)=\|X\|_{t}^{t}=\left|x_{1}\right|^{t}+\cdots+\left|x_{k}\right|^{t}(t>0)$, where $X=\left(x_{1}, \ldots, x_{k}\right)$ and $x_{i} \neq 0$. Then $f(t)=(g(t))^{1 / t}$.

Theorem 3. $f(t)$ is a monotone decreasing convex function and

$$
f^{\prime}(t)=\frac{f(t)}{t}\left(\frac{g^{\prime}(t)}{g(t)}-\ln f(t)\right) .
$$

Proof. It is easy to show that (7) holds. Without loss of generality, we assume that $\left|x_{1}\right| \leq\left|x_{2}\right| \leq \cdots \leq\left|x_{k}\right|$. Because

$$
\begin{aligned}
f^{\prime}(t) & =\frac{f(t)}{t}\left(\frac{g^{\prime}(t)}{g(t)}-\ln f(t)\right) \\
& =\frac{f(t)}{t^{2}}\left(\frac{\sum_{i=1}^{k}\left|x_{i}\right|^{t} \ln \left|x_{i}\right|^{t}}{g(t)}-\ln g(t)\right) \\
& \leq \frac{f(t)}{t^{2}}\left(\ln \left|x_{k}\right|^{t}-\ln g(t)\right) \leq 0,
\end{aligned}
$$

$f(t)$ is monotone decreasing.

Furthermore, $f(t)$ is a convex function. In fact, we have, by the convexity of function $f(x)=x^{2}$,

$$
\left(\frac{\sum_{i=1}^{k}\left|x_{i}\right|^{t} \ln \left|x_{i}\right|}{\sum_{i=1}^{k}\left|x_{i}\right|^{t}}\right)^{2} \leq \frac{\sum_{i=1}^{k}\left|x_{i}\right|^{t} \ln ^{2}\left|x_{i}\right|}{\sum_{i=1}^{k}\left|x_{i}\right|^{t}} .
$$

That is,

$$
\left(\frac{g^{\prime}(t)}{g(t)}\right)^{2} \leq \frac{g^{\prime \prime}(t)}{g(t)}
$$

Thus

$$
\left(\frac{g^{\prime}(t)}{g(t)}\right)^{\prime}=\frac{g^{\prime \prime}(t)}{g(t)}-\left(\frac{g^{\prime}(t)}{g(t)}\right)^{2} \geq 0
$$

and hence $g^{\prime}(t) / g(t)$ is monotone increasing. Since $f(t)$ is monotone decreasing, we know that $g^{\prime}(t) / g(t)-\ln f(t)$ is monotone increasing. Because $f(t) / t$ is monotone decreasing, $g^{\prime}(t) / g(t)$ is monotone increasing and $g^{\prime}(t) / g(t)-$ $\ln f(t) \leq 0$,

$$
\begin{aligned}
f^{\prime \prime}(t)= & \left(\frac{f(t)}{t}\right)^{\prime}\left(\frac{g^{\prime}(t)}{g(t)}-\ln f(t)\right) \\
& +\frac{f(t)}{t}\left(\frac{g^{\prime}(t)}{g(t)}-\ln f(t)\right)^{\prime} \geq 0
\end{aligned}
$$

which implies that $f(t)$ is convex function.

Theorem 4. For a given underdetermined linear system of equations $A_{m \times n} X=b$, there exists a constant $\gamma>0$ such that, for any $X_{1}, X_{2} \in \mathscr{X}$, either $f_{1}^{\prime}(t)=\left(\left\|X_{1}\right\|_{t}\right)^{\prime}<f_{2}^{\prime}(t)=$ $\left(\left\|X_{2}\right\|_{t}\right)^{\prime}$ or $f_{2}^{\prime}(t)=\left(\left\|X_{2}\right\|_{t}\right)^{\prime}<f_{1}^{\prime}(t)=\left(\left\|X_{1}\right\|_{t}\right)^{\prime}$ when $0<t<\gamma$.

Proof. Let $X^{k}=\left\{X \mid\|X\|_{0}=k, X \in \mathscr{X}\right\}$ and $X_{\beta}^{k}=\left\{X \in X^{k} \mid\right.$; there exists $\beta$ such that $\prod_{i=1}^{k}\left|x_{i}\right|=\beta$. Clearly, we have $X^{k}=$ $\cup_{\beta} X_{\beta}^{k}, \mathscr{X}=\cup_{k=1}^{n} X^{k}$.

Firstly, for any $X_{1}, X_{2} \in X_{\beta}^{k}$, there exists a constant $\gamma_{\beta}^{k}>0$ such that when $0<t<\gamma_{\beta}^{k}$, either $f_{1}^{\prime}(t)=\left(\left\|X_{1}\right\|_{t}\right)^{\prime}<f_{2}^{\prime}(t)=$ $\left(\left\|X_{2}\right\|_{t}\right)^{\prime}$ or $f_{2}^{\prime}(t)=\left(\left\|X_{2}\right\|_{t}\right)^{\prime}<f_{1}^{\prime}(t)=\left(\left\|X_{1}\right\|_{t}\right)^{\prime}$.

Obviously, for any given $X_{1}, X_{2} \in X_{\beta}^{k}$, there is a positive number $\left\{\gamma_{\beta}^{k}\right\}_{j}$ such that when $0<t<\left\{\gamma_{\beta}^{k}\right\}_{j}$, either $f_{1}^{\prime}(t)=$ $\left(\left\|X_{1}\right\|_{t}\right)^{\prime}<f_{2}^{\prime}(t)=\left(\left\|X_{2}\right\|_{t}\right)^{\prime}$ or $f_{2}^{\prime}(t)=\left(\left\|X_{2}\right\|_{t}\right)^{\prime}<f_{1}^{\prime}(t)=$ $\left(\left\|X_{1}\right\|_{t}\right)^{\prime}$. Hence, it suffices to show $\inf _{j}\left\{\gamma_{\beta}^{k}\right\}_{j}=\gamma_{\beta}^{k} \neq 0$. Otherwise, for an arbitrarily small positive number $\varepsilon$, there exists $t$ with $0<t<\varepsilon, Y_{1} \in X_{\beta}^{k}$, and $Y_{2} \in X_{\beta}^{k}$ such that

$$
f_{1}^{\prime}(t)=\left(\left\|Y_{1}\right\|_{t}\right)^{\prime}=f_{2}^{\prime}(t)=\left(\left\|Y_{2}\right\|_{t}\right)^{\prime} .
$$

Using (7) we obtain

$$
\begin{aligned}
& \frac{f_{1}(t)}{t}\left(\frac{g_{1}^{\prime}(t)}{g_{1}(t)}-\ln f_{1}(t)\right) \\
& =\frac{f_{2}(t)}{t}\left(\frac{g_{2}^{\prime}(t)}{g_{2}(t)}-\ln f_{2}(t)\right) .
\end{aligned}
$$

That is,

$$
\frac{g_{1}^{\prime}(t) / g_{1}(t)-\ln f_{1}(t)}{g_{2}^{\prime}(t) / g_{2}(t)-\ln f_{2}(t)}=\frac{f_{2}(t)}{f_{1}(t)} \text {. }
$$

Since $Y_{1}, Y_{2} \in X_{\beta}^{k}$, we have $\prod_{i=1}^{k}\left|y_{1 i}\right|=\prod_{i=1}^{k}\left|y_{2 i}\right|=\beta$.

Hence

$$
\sum_{i=1}^{k} \ln \left|y_{1 i}\right|=\sum_{i=1}^{k} \ln \left|y_{2 i}\right|
$$

Therefore, there is a positive integer $M$ such that

$$
\sum_{i=1}^{k} \ln ^{M}\left|y_{1 i}\right| \neq \sum_{i=1}^{k} \ln ^{M}\left|y_{2 i}\right|
$$


and, for any positive integer $N$ with $N<M$,

$$
\sum_{i=1}^{k} \ln ^{N}\left|y_{1 i}\right|=\sum_{i=1}^{k} \ln ^{N}\left|y_{2 i}\right|
$$

Since, for any positive integer $K$,

$$
\begin{aligned}
& g_{1}^{(K)}(0)=\left.\left(\left|y_{11}\right|^{t}+\cdots+\left|y_{1 k}\right|^{t}\right)^{(K)}\right|_{t=0}=\sum_{i=1}^{k} \ln ^{K}\left|y_{1 i}\right|, \\
& g_{2}^{(K)}(0)=\left.\left(\left|y_{21}\right|^{t}+\cdots+\left|y_{2 k}\right|^{t}\right)^{(K)}\right|_{t=0}=\sum_{i=1}^{k} \ln ^{K}\left|y_{2 i}\right|,
\end{aligned}
$$

$$
\begin{aligned}
\lim _{t \rightarrow 0}\left[\frac{g_{2}(t)}{g_{1}(t)}\right]^{1 / t^{M}} & =\exp \left\{\lim _{t \rightarrow 0} \frac{\ln g_{2}(t)-\ln g_{1}(t)}{t^{M}}\right\}=\exp \left\{\lim _{t \rightarrow 0} \frac{g_{2}^{\prime}(t) / g_{2}(t)-g_{1}^{\prime}(t) / g_{1}(t)}{M t^{M-1}}\right\} \\
& =\exp \left\{\lim _{t \rightarrow 0} \frac{g_{2}^{\prime \prime}(t) / g_{2}(t)-g_{1}^{\prime \prime}(t) / g_{1}(t)-g_{2}^{\prime 2}(t) / g_{2}^{2}(t)+g_{2}^{\prime 2}(t) / g_{1}^{2}(t)}{M t^{M-1}}\right\}=\cdots \\
& =\exp \left\{\frac{g_{2}^{(M)}(0)-g_{1}^{(M)}(0)}{k}\right\}>1 .
\end{aligned}
$$

And for the left of (21), we obtain

$$
\begin{aligned}
\lim _{t \rightarrow 0} & {\left[\frac{g_{1}^{\prime}(t) / g_{1}(t)-\ln f_{1}(t)}{g_{2}^{\prime} / g_{2}(t)-\ln f_{2}(t)}\right]^{1 / t^{M-1}} } \\
\quad & \exp \left\{\lim _{t \rightarrow 0} \frac{\ln \left(\ln g_{1}(t)-\left(g_{1}^{\prime}(t) / g_{1}(t)\right) \times t\right)-\ln \left(\ln g_{2}(t)-\left(g_{2}^{\prime}(t) / g_{2}(t)\right) \times t\right)}{t^{M-1}}\right\}=1 .
\end{aligned}
$$

This is a contradiction and thus when $0<t<\gamma_{\beta}^{k}$, either $f_{1}^{\prime}(t)=\left(\left\|X_{1}\right\|_{t}\right)^{\prime}<f_{2}^{\prime}(t)=\left(\left\|X_{2}\right\|_{t}\right)^{\prime}$ or $f_{2}^{\prime}(t)=\left(\left\|X_{2}\right\|_{t}\right)^{\prime}<$ $f_{1}^{\prime}(t)=\left(\left\|X_{1}\right\|_{t}\right)^{\prime}$.

Secondly, for any $X_{1}, X_{2} \in X^{k}$, there exists a constant $\gamma^{k}>$ 0 such that when $0<t<\gamma^{k}$, either $f_{1}^{\prime}(t)=\left(\left\|X_{1}\right\|_{t}\right)^{\prime}<f_{2}^{\prime}(t)=$ $\left(\left\|X_{2}\right\|_{t}\right)^{\prime}$ or $f_{2}^{\prime}(t)=\left(\left\|X_{2}\right\|_{t}\right)^{\prime}<f_{1}^{\prime}(t)=\left(\left\|X_{1}\right\|_{t}\right)^{\prime}$.

It suffices to show that $\inf _{\beta} \gamma_{\beta}^{k}=\gamma^{k} \neq 0$. Otherwise, for an arbitrarily small positive number $\varepsilon$, there is $t$ with $0<t<\varepsilon$, $Y_{1} \in X_{\beta_{1}}^{k}$ and $Y_{2} \in X_{\beta_{2}}^{k}\left(\beta_{1} \neq \beta_{2}\right)$ such that

$$
f_{1}^{\prime}(t)=\left(\left\|Y_{1}\right\|_{t}\right)^{\prime}=f_{2}^{\prime}(t)=\left(\left\|Y_{2}\right\|_{t}\right)^{\prime} .
$$

Using (7) again, we also obtain (15).
For the right of (15), we have

$$
\begin{aligned}
\lim _{t \rightarrow 0} \frac{f_{2}(t)}{f_{1}(t)} & =\exp \left\{\lim _{t \rightarrow 0} \frac{\ln g_{2}(t)-\ln g_{1}(t)}{t}\right\} \\
& =\frac{\prod_{i}\left|y_{1 i}\right|}{\prod_{i}\left|y_{2 i}\right|} \neq 1 .
\end{aligned}
$$

And for the left of (15), we have

$$
\begin{aligned}
\lim _{t \rightarrow 0} & \frac{g_{1}^{\prime}(t) / g_{1}(t)-\ln f_{1}(t)}{g_{2}^{\prime}(t) / g_{2}(t)-\ln f_{2}(t)} \\
= & \lim _{t \rightarrow 0} \frac{\left(g_{1}^{\prime}(t) / g_{1}(t)\right) \times t-\ln g_{1}(t)}{\left(g_{2}^{\prime}(t) / g_{2}(t)\right) \times t-\ln g_{2}(t)}=1 .
\end{aligned}
$$

This is a contradiction and thus $\inf _{\beta} \gamma_{\beta}^{k}=\gamma^{k} \neq 0$. 
Thirdly, for any $X_{1} \in X^{k}, X_{2} \in X^{s}, k \neq s$, there exists a constant $\gamma^{k, s}>0$ such that when $0<t<\gamma^{k, s}$, either $f_{1}^{\prime}(t)=$ $\left(\left\|X_{1}\right\|_{t}\right)^{\prime}<f_{2}^{\prime}(t)=\left(\left\|X_{2}\right\|_{t}\right)^{\prime}$ or $f_{2}^{\prime}(t)=\left(\left\|X_{2}\right\|_{t}\right)^{\prime}<f_{1}^{\prime}(t)=$ $\left(\left\|X_{1}\right\|_{t}\right)^{\prime}$.

We assume, without loss of generality, that $\left\|X_{1}\right\|_{0}=k<$ $s=\left\|X_{2}\right\|_{0}$. Then

$$
\begin{aligned}
& \lim _{t \rightarrow 0} \frac{g_{1}^{\prime}(t) / g_{1}(t)-\ln f_{1}(t)}{g_{2}^{\prime}(t) / g_{2}(t)-\ln f_{2}(t)} \\
& =\lim _{t \rightarrow 0} \frac{\left(g_{1}^{\prime}(t) / g_{1}(t)\right) \times t-\ln g_{1}(t)}{\left(g_{2}^{\prime}(t) / g_{2}(t)\right) \times t-\ln g_{2}(t)}=\frac{\ln k}{\ln s}<1, \\
& \lim _{t \rightarrow 0} \frac{f_{2}(t)}{f_{1}(t)}=\exp \left\{\lim _{t \rightarrow 0} \frac{\ln g_{2}(t)-\ln g_{1}(t)}{t}\right\}=\infty .
\end{aligned}
$$

So there is a positive number $\gamma^{k, s}$ such that when $t<\gamma^{k, s}$,

$$
\frac{g_{1}^{\prime}(t) / g_{1}(t)-\ln f_{1}(t)}{g_{2}^{\prime}(t) / g_{2}(t)-\ln f_{2}(t)}<\frac{f_{2}(t)}{f_{1}(t)}
$$

which implies that

$$
f_{2}^{\prime}(t)=\left(\left\|X_{2}\right\|_{t}\right)^{\prime}<f_{1}^{\prime}(t)=\left(\left\|X_{1}\right\|_{t}\right)^{\prime} .
$$

In conclusion, we take $\gamma=\min \left\{\gamma^{k}, \gamma^{k, s} \mid k, s=1,2, \ldots, n\right\}$ and thus when $0<t<\gamma$, for any $X_{1}, X_{2} \in \mathscr{X}$, either $f_{1}^{\prime}(t)=$ $\left(\left\|X_{1}\right\|_{t}\right)^{\prime}<f_{2}^{\prime}(t)=\left(\left\|X_{2}\right\|_{t}\right)^{\prime}$ or $f_{2}^{\prime}(t)=\left(\left\|X_{2}\right\|_{t}\right)^{\prime}<f_{1}^{\prime}(t)=$ $\left(\left\|X_{1}\right\|_{t}\right)^{\prime}$.

Obviously, for a given underdetermined linear system of equations $A_{m \times n} X=b$, there are infinitely many constants $\gamma_{i}>$ 0 such that when $0<t<\gamma_{i}$ Theorem 7 holds. The supremum of $\gamma_{i}$ is called the sparse constant of underdetermined linear system of equations $A_{m \times n} X=b$ and denoted $\gamma(A, b)$.

Corollary 5. Let equations $A_{m \times n} X=b$ be an underdetermined linear system. Then $f_{1}(t)=\left\|X_{1}\right\|_{t}$ and $f_{2}(t)=\left\|X_{2}\right\|_{t}$ have at most one intersection in $(0, \gamma(A, b))$, where $X_{1}, X_{2} \in X$ and $X_{1}$ is not the absolute value permutation of $X_{2}$.

Proof. It is easy to prove that the conclusion holds by Theorems 4 and 7.

Corollary 6. Let $X_{p}$ be the sparsest optimal solution to the problem $\left(P_{p}\right)(p<\gamma(A, b))$. Then $X_{p}$ is the unique sparsest optimal solution to the problem $\left(P_{p}\right)$ under the sense of absolute value permutation.

Proof. Suppose that $X_{p^{*}}$ is another sparsest optimal solution to the problem $\left(P_{p}\right)$ and $X_{p^{*}}$ is not the absolute value permutation of $X_{p}$. By Theorem 7, $\forall t \in(0, p)$, either $f_{1}^{\prime}(t)=$ $\left(\left\|X_{p}\right\|_{t}\right)^{\prime}<f_{2}^{\prime}(t)=\left(\left\|X_{p^{*}}\right\|_{t}\right)^{\prime}$ or $f_{1}^{\prime}(t)=\left(\left\|X_{p}\right\|_{t}\right)^{\prime}>f_{2}^{\prime}(t)=$ $\left(\left\|X_{p^{*}}\right\|_{t}\right)^{\prime}$. We suppose that $f_{1}^{\prime}(t)=\left(\left\|X_{p}\right\|_{t}\right)^{\prime}<f_{2}^{\prime}(t)=$ $\left(\left\|X_{p^{*}}\right\|_{t}\right)^{\prime}$ and hence $\forall t \in(0, p)$ we have $f_{1}(t)>f_{2}(t)$, which implies that $\left\|X_{p}\right\|_{0}>\left\|X_{p^{*}}\right\|_{0}$. This is a contradiction.
Theorem 7. The problem $\left(P_{p}\right)$ generates sparser solution as the value of $p$ decrease when $p<\gamma(A, b)$.

Proof. If the conclusion does not hold, then there exists the optimal solutions $X_{1}$ to the problems $\left(P_{p_{1}}\right)$ and the optimal solutions $X_{2}$ to the problems $\left(P_{p_{2}}\right)$ satisfying $p_{1}<p_{2}<$ $\gamma(A, b)$ and $\left\|X_{1}\right\|_{0}=s>k=\left\|X_{2}\right\|_{0}$. We consider the following two cases.

(1) If $\left\|X_{1}\right\|_{p_{1}}=\left\|X_{2}\right\|_{p_{1}}$, then $\left\|X_{1}\right\|_{p_{2}}<\left\|X_{2}\right\|_{p_{2}}$ because of Corollary 5 and $s>k$. This contradicts with the fact that $X_{2}$ is the optimal solutions to $\left(P_{p_{2}}\right)$.

(2) If $\left\|X_{1}\right\|_{p_{1}}<\left\|X_{2}\right\|_{p_{1}}$, then $\left\|X_{1}\right\|_{t}$ and $\left\|X_{2}\right\|_{t}$ have at least one intersection in $\left(0, p_{1}\right)$ because of $s>k$. Since $\left\|X_{2}\right\|_{p_{2}} \leq\left\|X_{1}\right\|_{p_{2}},\left\|X_{1}\right\|_{t}$, and $\left\|X_{2}\right\|_{t}$ have at least one intersection in $\left(p_{1}, p_{2}\right]$. This is contradictory to Corollary 5.

Theorem 8. Let $X_{1}$ and $X_{2}$ be the sparsest optimal solution to the problem $\left(P_{p_{1}}\right)$ and problem $\left(P_{p_{2}}\right)\left(p_{1}<p_{2}<\gamma(A, b)\right)$, respectively, and $X_{1}$ is not the absolute value permutation of $X_{2}$. Then there exist $t_{1}, t_{2} \in\left[p_{1}, p_{2}\right]$ such that when $p_{1} \leq t \leq$ $t_{1}, X_{1}$ is the sparsest optimal solution to the problem $\left(P_{t}\right)$ and when $t_{2}<t \leq p_{2}, X_{2}$ is the sparsest optimal solution to the problem $\left(P_{t}\right)$.

Proof. Firstly, $X_{1}$ is not the optimal solution to $P_{p_{2}}$ and hence $\left\|X_{1}\right\|_{p_{2}}>\left\|X_{2}\right\|_{p_{2}}$. In fact, if $\left\|X_{1}\right\|_{p_{2}}=\left\|X_{2}\right\|_{p_{2}}$, then $\left\|X_{1}\right\|_{p_{1}}<$ $\left\|X_{2}\right\|_{p_{1}}$ by Corollary 5 and $X_{1}$ is the optimal solution to the problem $\left(P_{p_{1}}\right)$. By Corollary 5 again, we have $\left\|X_{1}\right\|_{0}<\left\|X_{2}\right\|_{0}$ which contradicts with the fact that $X_{2}$ is the sparsest optimal solutions to $\left(P_{p_{2}}\right)$.

We consider the following two cases.

(1) If $\left\|X_{1}\right\|_{p_{1}}=\left\|X_{2}\right\|_{p_{1}}$, then, for any $p_{2} \geq t>p_{1}, X_{2}$ is the sparsest optimal solution to the problem $\left(P_{t}\right)$. Otherwise, there exists $X_{3}$ such that $\left\|X_{3}\right\|_{t}<\left\|X_{2}\right\|_{t}$ or $\left\|X_{3}\right\|_{t}=\left\|X_{2}\right\|_{t}$ and $\left\|X_{3}\right\|_{0}<\left\|X_{2}\right\|_{0}$. If $\left\|X_{3}\right\|_{t}<$ $\left\|X_{2}\right\|_{t}$, then $\left\|X_{3}\right\|_{0}>\left\|X_{2}\right\|_{0}$ by Corollary 5 and $\left\|X_{3}\right\|_{p_{1}} \geq\left\|X_{1}\right\|_{p_{1}}=\left\|X_{2}\right\|_{p_{1}}$, which is contradictory to Theorem 8. If $\left\|X_{3}\right\|_{t}=\left\|X_{2}\right\|_{t}$ and $\left\|X_{3}\right\|_{0}<\left\|X_{2}\right\|_{0}$, then $\left\|X_{3}\right\|_{p_{1}}<\left\|X_{2}\right\|_{p_{1}}=\left\|X_{1}\right\|_{p_{1}}$ by Corollary 5, which contradicts the fact that $X_{1}$ is the optimal solutions to $\left(P_{p_{1}}\right)$. Therefore, we pick $t_{1}=t_{2}=p_{1}$.

(2) If $\left\|X_{1}\right\|_{p_{1}}<\left\|X_{2}\right\|_{p_{1}}$, then, by $\left\|X_{1}\right\|_{p_{2}}>\left\|X_{2}\right\|_{p_{2}}$, $\left\|X_{1}\right\|_{t}$ and $\left\|X_{2}\right\|_{t}$ have one intersection $t_{0}$ in $\left(p_{1}, p_{2}\right)$, and hence $\left\|X_{1}\right\|_{0}<\left\|X_{2}\right\|_{0}$. We assume, without loss of generality, that $\left\|X_{1}\right\|_{0}+2=\left\|X_{2}\right\|_{0}$. Let $X_{3}$ be the sparsest optimal solution to the problem $P_{t_{0}}$. Then $X_{3}$ is not the absolute value permutation of $X_{2}$. Otherwise, we have $\left\|X_{3}\right\|_{t_{0}}=\left\|X_{2}\right\|_{t_{0}}=\left\|X_{1}\right\|_{t_{0}}$, that is, $X_{1}$ is the optimal solution to the problem $P_{t_{0}}$. Since $\left\|X_{1}\right\|_{p_{1}}<\left\|X_{2}\right\|_{p_{1}}=\left\|X_{3}\right\|_{p_{1}}$, we have $\left\|X_{1}\right\|_{0}<\left\|X_{3}\right\|_{0}$ which contradicts the fact that $X_{3}$ is the sparsest optimal solution to the problem $P_{t_{0}}$.

If $X_{3}$ is the absolute value permutation of $X_{1}$, then $\left\|X_{3}\right\|_{t_{0}}=\left\|X_{1}\right\|_{t_{0}}=\left\|X_{2}\right\|_{t_{0}}$ and thus, by the proof of case (1), 
for any $p_{2} \geq t>t_{0}, X_{2}$ is the sparsest optimal solution to the problem $\left(P_{t}\right)$. Obviously, for any $p_{1} \leq t \leq t_{0}, X_{1}$ is the sparsest optimal solution to the problem $\left(P_{t}\right)$. Therefore, we pick $t_{1}=t_{2}=t_{0}$.

If $X_{3}$ is not the absolute value permutation of $X_{1}$, then $\left\|X_{3}\right\|_{0}=\left\|X_{1}\right\|_{0}+1$ by Corollary 6 , and there exist $t_{1} \in\left(p_{1}, t_{0}\right)$, $t_{2} \in\left(t_{0}, p_{2}\right)$ such that $t_{1}$ is the intersection of $\left\|X_{3}\right\|_{t}$ and $\left\|X_{1}\right\|_{t}$ and $t_{2}$ is the intersection of $\left\|X_{3}\right\|_{t}$ and $\left\|X_{2}\right\|_{t}$. By the proof above, we have that, for any $t \leq t_{1}, X_{1}$ is the sparsest optimal solution to the problem $\left(P_{t}\right)$ and for any $t>t_{2}, X_{2}$ is the sparsest optimal solution to the problem $\left(P_{t}\right)$.

\section{Conclusion}

In this paper, the sparsity of underdetermined linear system via $l_{p}$ minimization for $0<p<1$ has been studied. Our research reveals that for a given underdetermined linear system of equations $A_{m \times n} X=b$ there exists a sparse constant $\gamma(A, b)>0$ such that when $p<\gamma(A, b)$, the problem $\left(P_{p}\right)$ generates sparser solution as the value of $p$ decreases and the sparsest optimal solution to the problem $\left(P_{p}\right)$ is unique under the sense of absolute value permutation and if $X_{1}$ is not the absolute value permutation of $X_{2}$ where $X_{1}$ and $X_{2}$ are the sparsest optimal solution to the problems $\left(P_{p_{1}}\right)$ and $\left(P_{p_{2}}\right)\left(p_{1}<p_{2}\right)$, respectively, then there exist $t_{1}, t_{2} \in\left[p_{1}, p_{2}\right]$ such that $X_{1}$ is the sparsest optimal solution to the problem $\left(P_{t}\right)\left(\forall t \in\left[p_{1}, t_{1}\right]\right)$ and $X_{2}$ is the the sparsest optimal solution to the problem $\left(P_{t}\right)\left(\forall t \in\left(t_{2}, p_{2}\right]\right)$.

\section{Conflict of Interests}

The authors declare that there is no conflict of interests regarding the publication of this paper.

\section{Acknowledgments}

This work was supported in part by the National Natural Science Foundation of China under Project 11271297, Project 11131006, Project 11201362, and EU FP7 Projects EYE2E (269118) and the National Basic Research Program of China under Project 2013CB329404.

\section{References}

[1] M.-J. Lai, "On sparse solutions of underdetermined linear systems," Journal of Concrete and Applicable Mathematics, vol. 8, no. 2, pp. 296-327, 2010.

[2] B. K. Natarajan, "Sparse approximate solutions to linear systems," SIAM Journal on Computing, vol. 24, no. 2, pp. 227-234, 1995.

[3] R. G. Baraniuk, "Compressive sensing," IEEE Signal Processing Magazine, vol. 24, no. 4, pp. 118-124, 2007.

[4] A. M. Bruckstein, D. L. Donoho, and M. Elad, "From sparse solutions of systems of equations to sparse modeling of signals and images," SIAM Review, vol. 51, no. 1, pp. 34-81, 2009.

[5] E. J. Candès, "Compressive sampling," in Proceedings of the International Congress of Mathematician, vol. 3, pp. 1433-1452, European Mathematical Society, Zürich, Switzerland, 2006.
[6] R. Gribonval and M. Nielsen, "Sparse representations in unions of bases," IEEE Transactions on Information Theory, vol. 49, no. 12, pp. 3320-3325, 2003.

[7] R. Chartrand, "Exact reconstruction of sparse signals via nonconvex minimization," IEEE Signal Processing Letters, vol. 14, no. 10, pp. 707-710, 2007.

[8] R. Chartrand and V. Staneva, "Restricted isometry properties and nonconvex compressive sensing," Inverse Problems, vol. 24, no. 3, Article ID 035020, pp. 1-14, 2008.

[9] X. Chen, F. Xu, and Y. Ye, "Lower bound theory of nonzero entries in solutions of $l_{2}-l_{q}$ minimization," SIAM Journal on Scientific Computing, vol. 32, no. 5, pp. 2832-2852, 2010.

[10] S. Foucart and M.-J. Lai, "Sparsest solutions of underdetermined linear systems via $l_{q}$-minimization for $0<q \leq 1$," Applied and Computational Harmonic Analysis, vol. 26, no. 3, pp. 395407, 2009.

[11] S. Foucart and M.-J. Lai, "Sparse recovery with pre-Gaussian random matrices," Studia Mathematica, vol. 200, no. 1, pp. 91$102,2010$.

[12] M.-J. Lai and J. Wang, "An unconstrained $l_{q}$ Minimization with $0 q \leq 1$ for sparse solution of underdetermined linear systems," SIAM Journal on Optimization, vol. 21, no. 1, pp. 82-101, 2011.

[13] Q. Sun, "Recovery of sparsest signals via $l_{q}$-minimization," Applied and Computational Harmonic Analysis, vol. 32, no. 3, pp. 329-341, 2012.

[14] Z. B. Xu, X. Chang, F. Xu, and H. Zhang, " $L_{1 / 2}$ regularization: a thresholding representation theory and a fast solver," IEEE Transactions on Neural Networks and Learning Systems, vol. 23, no. 7, pp. 1013-1027, 2012.

[15] Z. B. Xu, H. L. Guo, Y. Wang, and H. Zhang, "Representative of $L_{1 / 2}$ regularization among $L_{q}(0<q \leq 1)$ regularization: an experiments study based on phase diagram," Acta Automatica Sinica, vol. 38, no. 7, pp. 1225-1228, 2012.

[16] J. Zeng, S. Lin, Y. Wang, and Z. B. Xu, " $L_{1 / 2}$ regularization: convergence of iterative half thresholding algorithm," IEEE Transactions on Signal Processing, vol. 62, no. 9, pp. 2317-2329, 2014.

[17] M. Elad, Sparse and Redundant Representations: From Theory to Applications in Signal and Image Processing, Springer, New York, NY, USA, 2010. 


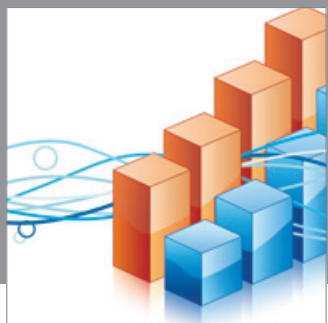

Advances in

Operations Research

mansans

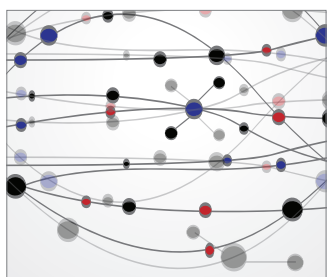

The Scientific World Journal
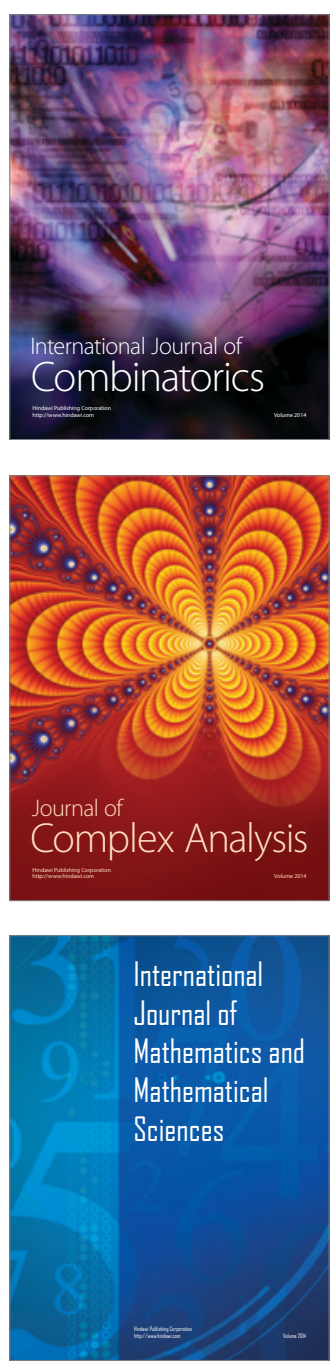
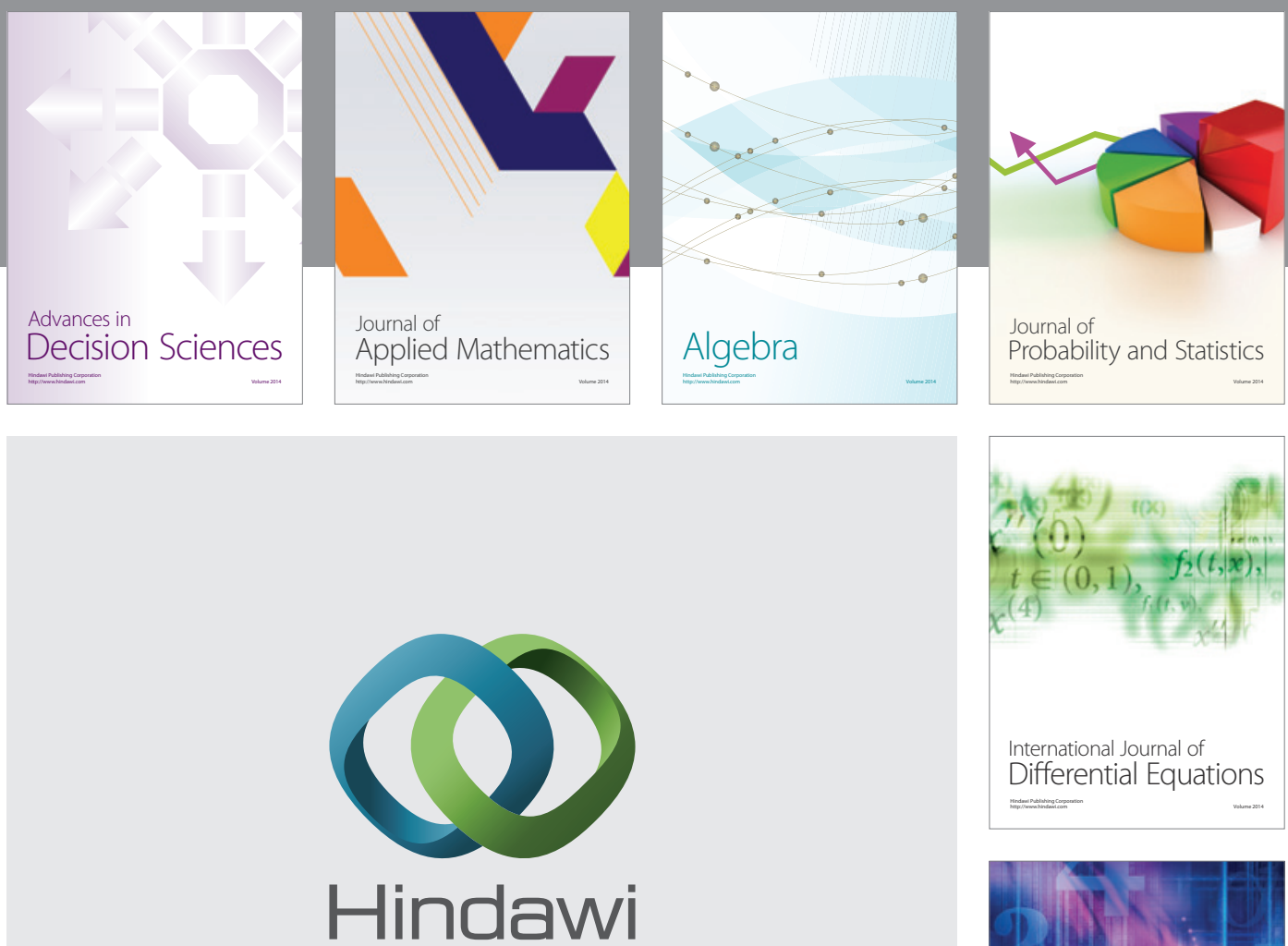

Submit your manuscripts at http://www.hindawi.com
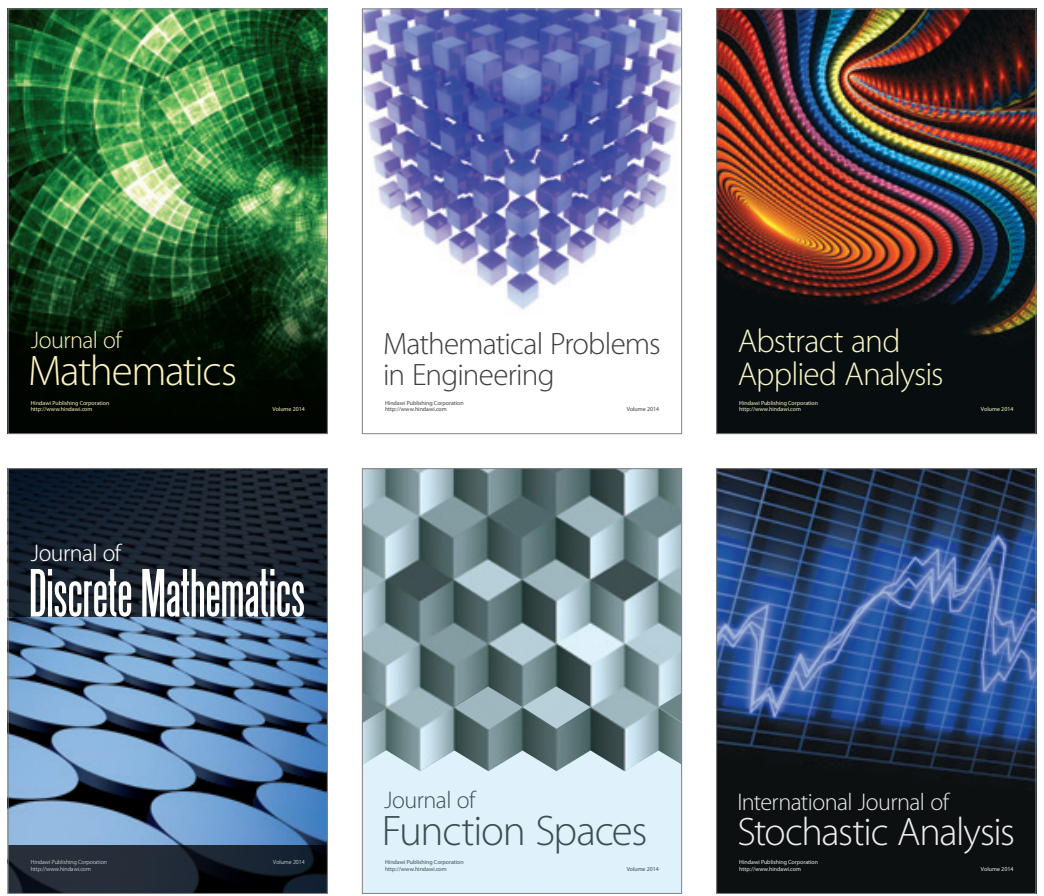

Journal of

Function Spaces

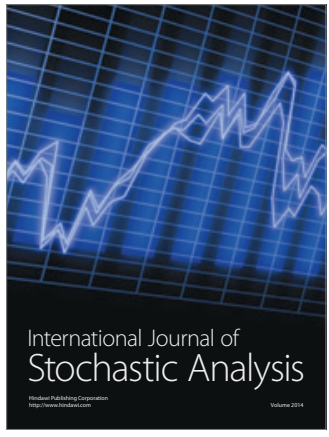

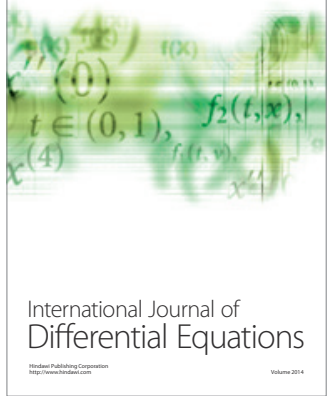
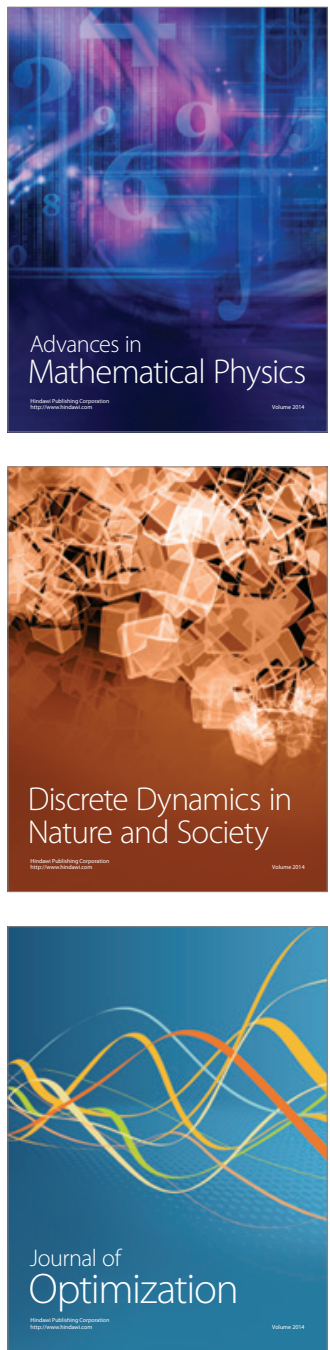